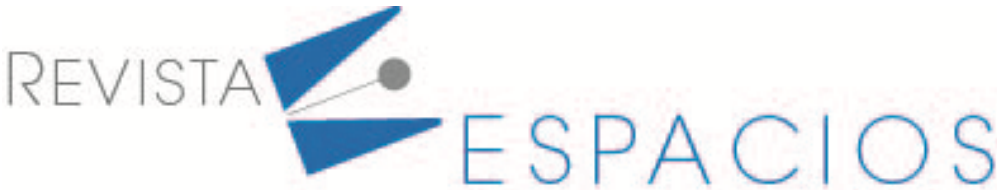

\section{Determinantes de la inversión extranjera directa en Latinoamérica (2000 - 2017)}

\section{Determinants of foreign direct investment in Latin America (2000 - 2017)}

\author{
CASTILLO, Erika E. ${ }^{1}$ \\ GONZÁLEZ, María G. ${ }^{2}$ \\ ZURITA, Eduardo G. ${ }^{3}$
}

\begin{abstract}
Resumen
El estudio establece los determinantes de la inversión extranjera directa (IED) en Latinoamérica. Se realizó un modelo econométrico por mínimos cuadrados generalizados, las variables explicativas fueron el producto interno bruto, la inflación, el desempleo y el ingreso neto bruto (INB) per cápita. Se encontró una relación directa entre la IED rezagada con la IED actual, así como con la tasa de crecimiento del PIB y el INB per cápita, mientras que las tasas de inflación y desempleo fueron no significativas.

Palabras clave: determinantes, inflación, inversión extranjera directa, producto interno bruto
\end{abstract}

\begin{abstract}
The study establishes the determinants of foreign direct investment (FDI) in Latin America, a generalized least squares econometric model was carried out, the explanatory variables were gross domestic product, inflation, unemployment and gross net income (GNI) per capita. A direct relationship was found between lagged FDI with current FDI and with the growth rate of GDP and GNI per capita, while inflation and unemployment rates were not significant.

Key words: determinants, inflation, foreign direct investment, gross domestic product
\end{abstract}

\section{Introducción}

La inversión extranjera directa (IED) es una partida positiva para las economías receptoras que se benefician por la colocación de capital en el país. Su estudio es importante porque con la inversión en los países beneficiarios se promueve la generación de fuentes de empleo, apertura mercados y se generan nuevas ideas, tecnologías y prácticas de trabajo, por lo que se considera favorable para el crecimiento económico de los países (Ormeño, Zambrano, \& Solorzano, 2011).

La entrada de IED en Latinoamérica está enfocada en los sectores de servicios y manufacturas, además los inversionistas son atraídos por los países que tienen recursos naturales en abundancia, tanto en el sector minero como en el de hidrocarburos, tal es el caso de Brasil, Colombia, Ecuador y Venezuela (Espín, Córdova, \& López, 2016). En Latinoamérica un factor muy importante para la IED es la ideología política ya que los países inversores

\footnotetext{
${ }^{1}$ Economista. Carrera de Economía. Facultad de Ciencias Políticas y Administrativas de la Universidad Nacional de Chimborazo, Ecuador. ecastillo.fpe@unach.edu.ec

${ }^{2}$ Economista y docente investigador. Facultad de Ciencias Políticas y Administrativas de la Universidad Nacional de Chimborazo, Ecuador. mggonzalez@unach.edu.ec

${ }^{3}$ Economista y docente investigador. Facultad de Ciencias Políticas y Administrativas de la Universidad Nacional de Chimborazo, Ecuador. ezurita@unach.edu.ec
} 
buscan estabilidad política para colocar su capital, esto les permitirá realizar inversiones a largo plazo, siendo beneficioso para ambas partes. Por otro lado, las políticas regulatorias del comercio internacional juegan un papel importante ya que estas pueden ser favorables o no para los países inversores (Goto, Lagos , \& Spencer, 2016).

Según la CEPAL (2007), entre los años 1996 y 2001 los flujos de IED en América Latina fueron mayores al 4\% del PIB. Para el año 2007 este porcentaje se incrementó en 8\% de promedio hasta llegar al 12\% del PIB; mientras que en 2011, aun afectada por la crisis económica, la IED representó el 5,8\% del PIB. Por otra parte, la CEPAL (2016) estima que entre el 2003 y 2013 la inversión extranjera directa pasó de 46,937 millones de dólares a un récord de 189,951 millones de dólares. Países desarrollados de Europa, Asia y América del Norte han visto a América Latina como una región atractiva para la implementación de estrategias para la dinamización económica, lo que ha permitido que la IED crezca no solo en el sector de recursos naturales, sino que también en los relacionados a los servicios financieros y de telecomunicaciones (Penfold \& Curbelo, 2013).

En América Latina y el Caribe en el año 2013 los flujos de IED alcanzaron los 189.951 millones de dólares, con una tasa de crecimiento del $8,8 \%$ con respecto a 2012. Siendo este rubro el más alto en el período de estudio. Para el año 2014 el flujo de IED llegó a 158.803 millones de dólares lo cual representó una caída de 16,4\% en comparación a 2013 (Goto, Lagos , \& Spencer, 2016). La inversión extranjera directa en la región aumentó en el año 2015 a 179.100 millones de dólares; sin embargo para los años 2016 y 2017 disminuyó a 167.189 y 161.911 millones de dólares respectivamente (CEPAL, 2018).

\subsection{Antecedentes}

En el estudio de los factores determinantes de la IED y su incidencia en el crecimiento económico y la capacidad de generación de riqueza en los países receptores, basado en la teoría del enfoque ecléctico y la teoría de Ozawa y mediante un análisis empírico utilizando el modelo econométrico de regresión lineal múltiple y efectos fijosaleatorios mediante datos de panel, se concluye que la inflación en los países juega un papel negativo ya que esta perjudica al crecimiento productivo, el consumo y por ende al empleo, por otra parte, se considera que el crecimiento del PIB incide de forma positiva en la recepción de IED en la región, ya que esta contribuye al crecimiento económico de los países y es un punto atractivo para los inversionistas; entre los ejemplos más representativos están países como: Brasil, Colombia, Perú y Uruguay; ya que al controlar en la última década los índices de inflación y mejorar el crecimiento del PIB, tuvieron una mayor recepción de IED (Gil , López, \& Espinosa, 2013).

En el análisis de los factores determinantes de la inversión extranjera directa y las características que explican el escenario de los países con menores avances, con base en la teoría del paradigma de OLI y realizando un estudio econométrico de mínimos cuadrados generalizados, los resultados obtenidos muestran que la IED en los países de la región está determinada por el tamaño de mercado y la apertura comercial ya que estos factores son parte fundamental de la dinamización económica, por otra parte, el riesgo país y las privatizaciones son determinantes negativos, ya que estos crean incertidumbre en los inversionistas. También los resultados confirman que los costes laborables, no reflejan mayor importancia para la atracción de la inversión extranjera (Mogrovejo, 2005).

En el estudio de los factores determinantes de la inversión extranjera directa y su incidencia en las economías receptoras mediante una revisión de literatura se concluye que la inversión extranjera vertical como en la horizontal los inversionistas buscan invertir en países que tengan un ambiente político que sea estable y seguro, ya que les permitirá realizar inversiones de largo plazo, y estos producen costos fijos los cuales también serán recuperados al largo plazo, por tanto, evitan invertir en regiones con una alta inestabilidad política donde pueden correr el riesgo de ser expropiados. A su vez los inversionistas desean encontrar un sistema legal transparente 
donde se respete la propiedad intelectual, ya que esto les permitirá a no estar expuestos a competencias desleales (Jiménez \& Rendón, 2012).

Con base en la observación de los indicadores económicos de los 4 países de América Latina con mayor ingreso de IED, se analiza en qué medida los determinantes de inversión extranjera directa influyen en los niveles de inversión realizado por empresas extranjeras en Latinoamérica, en donde se pudo apreciar que hay una relación entre los determinantes de inversión que utilizan los países a nivel mundial y los niveles de IED al interior de los distintos sectores de los países de Latinoamérica. En ese sentido, problemas como la corrupción y la inestabilidad política, afectan negativamente en el nivel de IED que recibe el país, debido al riesgo que este puede representar al país inversor. Por otro lado, se logró ver que ciertas diferencias entre los factores, pueden inferir al momento de realizar la colocación de capitales, esto se debe principalmente a que las empresas con un enfoque global se basan en la eficiencia de las empresas, por lo que el costo bajo de mano de obra, a recursos naturales no explotados, a la posesión de mercados que cuenten con infraestructura para la producción a gran escala, forman parte de factores clave al momento de decidir dónde invertir (Goto, Lagos , \& Spencer, 2016).

Mediante un análisis de datos de panel de efectos fijos y aleatorios, realizado a 22 países de Latinoamérica, considerando variables macroeconómicas y aspectos políticos, se pudo comprobar que la variable riesgo país y el índice de libertad económica son factores determinantes que influyen en los flujos de inversión, los autores afirman que para los inversionistas es importante tener un ambiente de seguridad, estabilidad económica, política y laboral, tratando de obtener seguridad en sus inversiones, a su vez se debe fomentar el comercio exterior y la eliminación de la corrupción. En el estudio se toma en consideración variables cualitativas, como la ideología política ya que esto incide de forma positiva o negativa en el momento de realizar la inversión, tal es el caso de los países que conforman el ALBA, ya que tienen una ideología izquierdista por tal motivo tiene una relación negativa en la aplicación de IED (Ormeño, Zambrano, \& Solorzano, 2011).

\subsection{Inversión Extranjera Directa}

El Banco Mundial (2009), indica que la inversión extranjera directa significa "Adquirir intereses de largo plazo en una empresa que esté operando en otro país diferente al del inversor, el propósito del inversor es el de tener una voz participativa en el manejo de dicha empresa en el extranjero" (p.34).

Por otra parte, el Observatorio de Multinacionales de América Latina (2012) señala que la inversión extranjera directa es la inversión de capital por parte de una persona natural o de una persona jurídica en un país distinto al de origen. En el país beneficiario, estas inversiones pueden colocarse mediante la creación de nuevas industrias o la inversión en empresas ya posesionadas.

\subsection{Tipos de la Inversión Extranjera Directa}

Chávez y Félix (2003) afirman que la inversión extranjera directa se ve motivada por dos tipos tanto por la IED Horizontal como la IED Vertical, tal como se describe a continuación.

\subsubsection{Inversión Extranjera Directa Horizontal}

Para Cabrera y Naranjo (2018), la IED horizontal son las actividades económicas que se realizan en otros países con el objetivo principal de producir más cerca de los consumidores con el fin de reducir costos de transporte. Por otra parte, para Chávez y Félix (2003) consideran que la IED horizontal se refiere a la existencia de una inversión de la misma industria pero en otro país, es decir como cuando se abre una sucursal en el exterior.

\subsubsection{Inversión Extranjera Directa Vertical}

Cabrera y Naranjo (2018), señalan que la IED vertical divide las actividades de una empresa geográficamente por función. Es decir, que las empresas inversionistas buscan minimizar costos en países donde se puede desarrollar 
los procesos productivos a bajos costes, es decir las actividades intelectuales se realizan en la matriz mientras que los procesos de producción en países con mano de obra calificada y con menores costes de mano de obra. Por otra parte, Saravia (2009) menciona que la IED vertical corresponde a las categorías de búsqueda de recursos, eficiencia y, en algunos casos, de activos. Los modelos de IED vertical habitualmente se caracterizan por tener una empresa como casa matriz y una planta de producción, ubicada en un espacio geográfico distinto.

\subsection{Teorías de la Inversión Extranjera Directa}

Díaz (2003) señala diversas teorías que explican los factores determinantes de la IED.

\section{Enfoque Ecléctico o Paradigma OLI}

Dunning (1977), como se lo citó en el trabajo de Díaz (2003), desarrolla el enfoque ecléctico o "paradigma OLI", en donde busca definir cuáles son los factores y condicionantes que determinan la localización de las principales actividades económicas positivas que atraen a las empresas a realizar inversiones. Las siglas OLI se refieren a ciertas ventajas como la propiedad de la empresa, la internalización del proceso productivo y la localización de los países de destino de la IED. Las ventajas de propiedad de la empresa y la internacionalización del proceso productivo justifican la presencia de actividades de IED en otros países, en donde se toma en consideración activos intangibles como la marca, tecnología y gestión empresarial de la empresa que se desea explotar en el mercado exterior, a su vez deben tener en cuenta características muy importantes como ventajas o inputs, las cuales deben dar mayor beneficio al que podrían alcanzar al posesionar la empresa en su propia nación. Las ventajas de localización hacen alusión a los costes y a los factores de producción disponibles, en donde buscan recursos naturales, mercados nuevos, eficiencia y estabilidad económica, a la vez se toma en cuenta el sistema institucional vigente, la intervención del gobierno en el sector económico, y la presencia de economías de escala.

\section{Teoría del ciclo de vida del producto de Vernon}

La teoría formulada por Vernon (1966), en un intento de romper con las teorías tradicionales del comercio internacional y de localización de la IED ya que en estas los costes son tomados como factores no tan relevantes, afirma que las empresas inversoras buscan mayores beneficios en los países menos desarrollados ya que estos tienen menores costes laborales y de producción, considerando que este punto es el de mayor importancia al momento de invertir en otras economías con el objetivo de incentivar la producción de bienes que estén ligados a empresas monopólicas, en donde se busca un valor agregado para dichos bienes para que así sean más atractivos y se puedan ofertar en países subdesarrollados, incentivando de esta manera la dinamización de la economía de los países receptores de IED (Díaz, 2003).

\section{Teoría de Ozawa}

Díaz (2003) analiza la teoría dinámica desarrollada por Ozawa, y explica que el impacto de la IED en el desarrollo económico de los países receptores, el estudio se profundiza en los factores determinantes que pueden establecer la localización de los flujos de IED, esta colocación de capital seria atraídos por economías menos desarrolladas, las cuales contribuyen a un cambio estructural ayudando al incremento de la renta y evolucionando la composición de la demanda nacional, dando inicio a una nueva IED la cual es denominada "market seeking", la cual consiste en mitigar costes de transporte, costes salariales, evitar barreras comerciales y buscar países con abundancia de recursos no explotados.

\subsection{Determinantes de la Inversión Extranjera Directa}

Para Flora y Agrawal (2014) señalan que los flujos de inversión extranjera directa están determinados por factores económicos, políticos, socio-económicos y científicos, tal como se muestra en la tabla 1. 
Tabla 1

Determinantes de la inversión extranjera

\begin{tabular}{|c|c|}
\hline \multirow{10}{*}{$\begin{array}{l}\text { Determinantes } \\
\text { económicos }\end{array}$} & $\begin{array}{l}\text { (+) Tamaño de mercado: las empresas buscan beneficiarse de la economía de los países ya sea } \\
\text { por la oportunidad de venta o el nivel de producción, la cual se mide a través del PIB, PIB per } \\
\text { cápita, PNB per cápita y población. }\end{array}$ \\
\hline & $\begin{array}{l}\text { (+) Tasa de crecimiento del mercado: se observa la dinamización económica y desarrollo de un } \\
\text { país, se mide a través de la tasa de crecimiento del PIB. }\end{array}$ \\
\hline & (-) Inflación: determina la estabilidad macroeconómica de un país. \\
\hline & $\begin{array}{l}\text { ( } \pm \text { )Tasa de cambio: determina como la apreciación de la moneda puede influir en los flujos de } \\
\text { IED. }\end{array}$ \\
\hline & $\begin{array}{l}\text { (+) Crecimiento del dinero: se refiere a la cantidad de dinero que existe en una economía y del } \\
\text { desarrollo y crecimiento del sistema financiero de un país. }\end{array}$ \\
\hline & $\begin{array}{l}\text { (+) Apertura de intercambio: es fundamental para las economías ya que es un impulsador para } \\
\text { el crecimiento, es decir que a mayor apertura comercial, la colocación de flujos de IED serán } \\
\text { mayores. }\end{array}$ \\
\hline & $\begin{array}{l}\text { ( } \pm \text { )Infraestructura: la instalación de infraestructura es fundamental, ya que es un limitante para } \\
\text { los flujos de IED, es decir que si un país no cuenta con fuentes de luz, agua potable, movilidad, } \\
\text { telecomunicaciones, entre otros, es más probable a que no reciba flujos de capital. }\end{array}$ \\
\hline & $\begin{array}{l}\text { ( } \pm \text { )Tasa de interés: se refiere al costo de capital, por lo cual los inversionistas se ven atraídos } \\
\text { cuando hay tasas de interés bajas. }\end{array}$ \\
\hline & $\begin{array}{l}\text { (+) Tasa de retorno sobre la inversión: el inversor evalúa los mercados para determinar cuál será } \\
\text { el rendimiento de su inversión. }\end{array}$ \\
\hline & $\begin{array}{l}\text { (-) Déficit de la cuenta corriente: el saldo de la cuenta corriente es un factor importante al } \\
\text { momento de invertir, es decir que si existe un déficit en la cuenta este país no será atractivo para } \\
\text { la colocación de capitales. }\end{array}$ \\
\hline \multirow{3}{*}{$\begin{array}{l}\text { Determinantes } \\
\text { políticos }\end{array}$} & $\begin{array}{l}\text { (-) Impuestos: en un país que tenga mayor cantidad de impuestos, este no será atractivo para los } \\
\text { inversionistas, ya que esto representa un mayor costo al momento de invertir. }\end{array}$ \\
\hline & $\begin{array}{l}\text { ( } \pm \text { ) Estabilidad política: actúa como un instrumento impulsador de la IED, es decir que al existir } \\
\text { incertidumbre o crisis políticas, los flujos de IED serán menores. }\end{array}$ \\
\hline & $\begin{array}{l}\text { (+) Política de gobierno: es un factor fundamental para la atracción de IED, ya que mediante } \\
\text { políticas macroeconómicas, de incentivos, exenciones fiscales, subsidios, entre otros, los } \\
\text { inversionistas se ven atraídos para color capitales en estos países. }\end{array}$ \\
\hline \multirow{4}{*}{$\begin{array}{l}\text { Determinantes } \\
\text { socio-económicos }\end{array}$} & $\begin{array}{l}\text { (+) Capital humano: es un factor que puede ser muy importante al momento de colocar flujos } \\
\text { de capital, ya que si un país cuenta con mano de obra calificada y mejor nivel de educación que } \\
\text { impulse a mayores niveles de producción, los inversionistas se verán atraídos por este. }\end{array}$ \\
\hline & $\begin{array}{l}\text { (+) Recursos naturales: el inversor se ve atraído cuando en los países receptores se cuenta con } \\
\text { recursos naturales (minería, petróleo, productos agrícolas), para poder explotarlos y beneficiarse } \\
\text { de ello. }\end{array}$ \\
\hline & $\begin{array}{l}\text { (-) Nivel de corrupción: la incertidumbre, la falta de transparencia y de información, es un punto } \\
\text { negativo para la colocación de inversiones, ya que esto representa riesgos que debe tomar los } \\
\text { inversionistas al momento de invertir. }\end{array}$ \\
\hline & $\begin{array}{l}\text { (+) Integración regional: el comercio y los acuerdos existentes con otros países son muy } \\
\text { importantes, puesto que, la reducción de barreras arancelarias y reducción de costos comerciales } \\
\text { son incentivos para los inversionistas. }\end{array}$ \\
\hline \multirow{2}{*}{$\begin{array}{l}\text { Determinantes } \\
\text { científicos }\end{array}$} & $\begin{array}{l}\text { (+) Investigación y desarrollo (I+D): la investigación y el desarrollo es una fuente positiva al } \\
\text { momento de atraer IED, ya que esto reduce el costo al momento de adquirir mano de obra } \\
\text { calificada. }\end{array}$ \\
\hline & $\begin{array}{l}\text { (+) Avances tecnológicos: los avances tecnológicos y de innovación, permiten que exista mayor } \\
\text { desarrollo lo cual es atractivo para los inversionistas. }\end{array}$ \\
\hline
\end{tabular}

Fuente: Elaboración propia con base al trabajo de Flora y Agrawal (2014)

Los inversores a través de sus empresas internacionales, buscan rentabilidad y estabilidad económica para así aumentar sus ganancias y que su posicionamiento a nivel global sea más fuerte, por lo cual toman en cuenta distintos determinantes al momento de invertir sus capitales, por tal motivo en la tabla 1 se ha clasificado los 
determinantes que influyen positivamente en la IED como el tamaño de mercado, tasa de crecimiento del mercado, crecimiento del dinero, apertura de intercambio, tasa de retorno sobre la inversión, política de gobierno, capital humano, recursos naturales, integración regional, investigación y desarrollo, y avances tecnológicos, por lo que ante un incremento de los factores antes enunciados se promueve la IED, mientras que los factores que influyen negativamente son la inflación, déficit de la cuenta corriente, impuestos, nivel de corrupción, éstos muestran una relación inversa, es decir que ante un incremento de dichos factores la IED se contrae; tomando en cuenta estos aspectos los países deben aplicar políticas que incentiven a la colocación de capitales ya que estos flujos son de gran importancia ya que promueven la dinamización económica y social (Flora y Agrawal, 2014).

\section{Metodología}

La investigación inicia con la observación y descubrimiento del problema, a su vez se aplica los métodos analítico, hipotético-deductivo e histórico, los cuales permiten conocer el problema y analizar las variables a estudiar. La investigación es de carácter descriptiva, explicativa y correlacional en la cual se explica y se describe el comportamiento de las distintas variables, y mediante un modelo econométrico se muestra la relación existente entre estas.

Posteriormente se creó una base de datos de las variables para el período de estudio 2000 - 2017, que se extrajo de fuentes secundarias como informes anuales del Banco Mundial y de la CEPAL para analizar el comportamiento de la inversión extranjera directa y sus factores determinantes. Mediante la aplicación de un modelo econométrico de mínimos cuadros generalizados, usando datos de panel para los 19 países latinoamericanos en el período de estudio, se determinó la relación existente entre las mismas para comprobar la hipótesis, teniendo como referencia el trabajo de Gil, López y Espinosa (2013) y de Mogrovejo (2005) en los cuales se emplean modelos de mínimos cuadrados generalizados y modelos estáticos: efectos fijos y efectos aleatorios, la representación matemática es la siguiente:

$Y_{i t}=A_{1} Y_{i t-1}+\beta_{0}+\beta_{1} X_{1 i t}+\beta_{2} X_{2 i t}+\cdots+\mu_{i t}$

Dónde:

$Y_{i t}=$ Vector de variable endógena

$X_{1 i t}=$ Vector de variables exógenas

A $y \beta=$ Matrices de coeficientes

$\mu_{i t}=$ Término de perturbación estocástico para t períodos.

El procedimiento inicia con la estimación econométrica mediante la aplicación de una regresión de mínimos cuadrados generalizados (MCG), el cual se utiliza para identificar si las variables exógenas son significativas para el modelo aplicado, es decir permite comprobar si las variables utilizadas son determinantes de la inversión extranjera directa en Latinoamérica durante el periodo de estudio. Continuando con la aplicación del Test de Hausman el cual permite establecer si existe diferencias significativas en las medias de las variables utilizadas en los modelos estáticos aplicados, lo cual permitirá conocer si se debe aplicar el modelo de efectos fijos o efectos variables. Con base al análisis anterior se procede a estimar el método de MCG con el modelo estático que sea óptimo para el modelo econométrico.

El análisis de datos de los factores determinantes de la inversión Extranjera Directa se desarrolla con base en la siguiente expresión:

$\log Y_{i t}=a_{1} \log Y_{i t-1}+\beta_{0}+\beta_{1} \log X_{1 i t}+\beta_{2} \log X_{2 i t}+\beta_{3} \log X_{3 i t}+\beta_{4} \log X_{4 i t}+\mu_{i t}$ 
Dónde:

$\log Y_{i t}=$ Es el logaritmo de la inversión extranjera directa como entrada neta de capital en los diferentes países, la cual fue obtenida del Banco Mundial. Siendo de importancia su estudio ya que es la interacción entre los países mediante el comercio, ayudando de forma positiva al crecimiento económico y por ende al desarrollo económico y social de un país (Rivas y Puebla, 2016).

$a_{1} \log Y_{i t-1}=$ es el logaritmo de la inversión extranjera directa rezagada en un periodo de tiempo, ya que si un país tiene captación de inversión positiva para los años siguientes este comportamiento se mantendrá positivo y viceversa.

$\log X_{1 i t}=$ es el logaritmo de la tasa de crecimiento del producto interno bruto en porcentajes anuales, obtenidos del Banco Mundial. Este indicador ayuda a medir el tamaño de mercado de un país el cual representa la riqueza del mismo, es decir que si un país tiene niveles de producción altos este influirá positivamente a la IED.

$\log X_{2 i t}=$ es el logaritmo de la tasa de inflación en porcentajes anuales, obtenidos del Banco Mundial. Es un indicador que refleja el aumento porcentual de los precios de bienes y servicios en un periodo de tiempo, el cual incide negativamente a los flujos de IED, ya que esta variable representa la estabilidad económica de un país, es decir si un país tiene niveles altos de inflación este no será atractivo para los inversionistas.

$\log X_{3 i t}=$ es el logaritmo de la tasa de desempleo en porcentajes anuales de la población activa total, obtenido del Banco Mundial. Es un indicador que mide el nivel de desempleo en un país el cual tiene una relación indirecta con la IED ya que a mayor desempleo la IED se verá afectada negativamente.

$\log X_{4 i t}=$ es el logaritmo del ingreso nacional bruto per cápita en precios constantes (método Atlas), obtenidos del Banco Mundial. Este indicador mide el valor de los bienes y servicios producidos por los residentes de un país en un periodo de tiempo dividido para la población total, siendo un indicador positivo para la IED, ya que a mayor INB per cápita mayor será la colocación de capital en los países.

$\mu_{i t}=$ Término de perturbación estocástico para t períodos.

\section{Resultados}

\subsection{Inversión Extranjera Directa}

Para América Latina la inversión extranjera directa ha adquirido un papel importante, ya que en los últimos años los niveles de esta han crecido notablemente, lo cual ayudó a la dinamización de la economía en la región, especialmente en países ricos de recursos naturales (CEPAL, 2018). Es así, que en el Gráfico 1 se observa el comportamiento de la inversión extranjera directa en la región de Latinoamérica, en donde existen fluctuaciones tanto crecientes como decrecientes. 


\section{Gráfico 1}

Inversión Extranjera Directa en países de Latinoamérica

Período 2000 - 2017, (miles de millones de dólares, valores promedios y porcentajes)

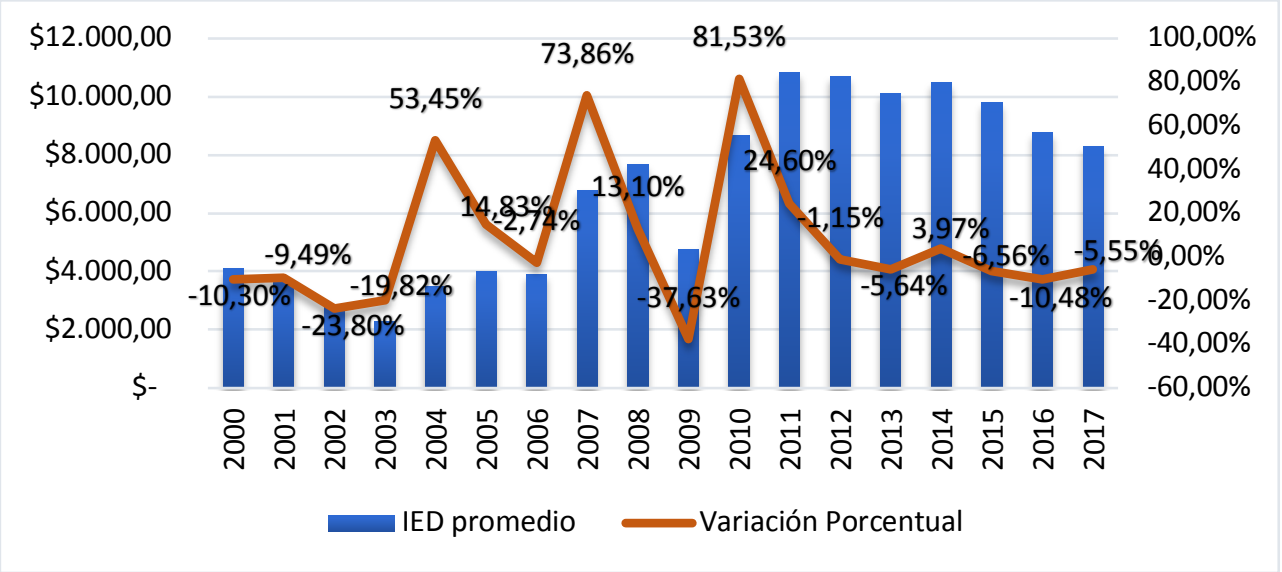

Fuente: Elaboración propia con base en datos del Banco Mundial (2019)

Durante el período 2000-2003 la tasa de variación promedio fuede-15,85\%, esto se dio por la crisis política y económica en Argentina y la incertidumbre de que esta afectaría a toda la región. Por otro lado, la caída de los precios de las acciones de las empresas privadas en la región y el aumento a la salida de utilidades y recursos derivados a la IED, fueron motivo para la caída de la inversión en el periodo antes mencionado (CEPAL, 2004).

Para el año 2004 los flujos de IED ascendieron en 53,45\% con respecto al año 2003, estos flujos se vieron motivados por el sector manufacturero, el sector de servicios y en especial por el sector de recursos naturales los cuales estaban en manos de empresas privadas, siendo atractivo para inversionistas europeos. Este crecimiento en los flujos se mantuvo hasta el año 2008 , con una variación porcentual del $30 \%$ durante el período 2004 - 2008, lo cual fue de optimismo para la región, esto se debió a la estabilidad económica y al aumento de la demanda mundial en la región(CEPAL, 2009). Mientras que para el año 2009 los flujos de IED en Latinoamérica disminuyeron en $37,63 \%$ a causa de la crisis mundial ya que esta incidió directamente en la producción y en el comercio internacional causado por la desaceleración económica de los países desarrollados, siendo estos los principales inversionistas y afectando directamente a México (CEPAL, 2010).

Para el año 2010 la IED aumentó en 81,53\% ya que existió una recuperación de las economías desarrolladas, a su vez se impulsaron ciertos sectores, tales como el sector de recursos naturales, sector manufacturero y el sector automotriz. Por otra parte, el aumento de la demanda interna en ciertos países ha sido una parte fundamental para la atracción de inversionistas, lo cual ha motivado un crecimiento en la IED hasta el año 2011(CEPAL, 2012).Para el año 2012 se reflejó un decrecimiento del 1,15\%, debido al bajo flujo de IED en el mundo, lo cual disminuyó la colocación de inversiones en los principales países de Latinoamérica, a su vez esta reducción de IED se vio afectado también por la caída del 34,9\% en México a causa de que el sector financiero se vio afectado por la venta de acciones del Banco Santander México siendo uno de los principales entes financieros en dicho país (Gobierno de México, 2013).Esta reducción de IED se mantuvo por el siguiente año, ya que la volatilidad y la incertidumbre de los mercados financieros en Estados Unidos, llevo a la Reserva Federal a tomar la decisión de revertir la política de flexibilidad cuantitativa que redujo la rentabilidad de Estados Unidos, siendo esta decisión un riesgo en las economías latinoamericanas principalmente en los países con mayor colocación de capitales (CEPAL, 2014).

En el año 2014 la IED tuvo un crecimiento del 3,97\%, motivo del incremento de inversiones mundiales en países en desarrollo de América Latina (CEPAL, 2015). Para el año 2015 existió una reducción del 6,56\% en la IED a causa de la disminución de inversiones en sectores naturales, tal es el caso del sector minero en consecuencia a la caída 
de los precios de los minerales, afectando de forma directa a Colombia y Chile, por otra parte, a causa de la desaceleración económica que sufrieron ciertos países de América Latina principalmente en Brasil, dando como resultado un decrecimiento del $23 \%$ en las inversiones de dicho país afectando notablemente a la IED de todo América Latina (CEPAL, 2016).

Los flujos de IED para el año 2016 decrecieron en un 10,48\% en consecuencia a las afectaciones del año anterior, al igual que para el año 2017 con una caída del 5,55\% por tercer año consecutivo, afectado por la caída en los precios de los productos básicos de exportación y a su vez la caída de los prestamos entre compañías en Brasil, es decir una caída en el sector financiero hizo que se presente un decrecimiento del 9\% en la IED del país, por otro lado, Chile presentó una reducción del48\%en la IED a causa de la caída del precio del cobre desde el año 2011 al 2016, esto es perjudicial a la IED de América Latina ya que estos países son los dos principales receptores de inversiones, para el resto de los países de latinoamericanos los flujos de IED aumentaron en porcentajes mínimos (CEPAL, 2018).

\subsection{Tasa de Crecimiento del PIB}

La tasa de crecimiento del PIB en América Latina es muy importante ya que de esta depende el crecimiento económico de los países y por ende de su desarrollo, en donde durante el período de estudio la tasa de crecimiento del PIB ha sido muy volátil debido a factores internos y externos.

\section{Gráfico 2}

Evolución del PIB de

Latinoamérica (19 países)

Período 2000 - 2017, (miles de millones de dólares, valores promedios y porcentajes)

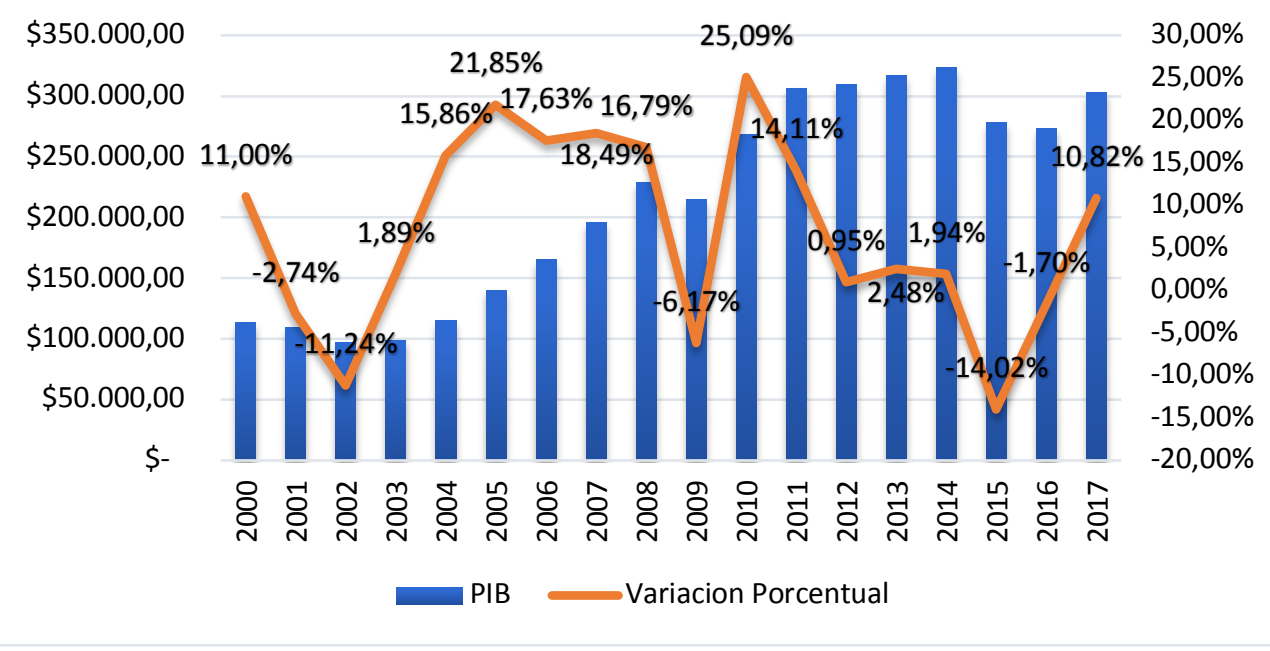

Fuente: Elaboración propia con base en datos del Banco Mundial (2019)

Se puede observaren el Gráfico 2 que la tasa de crecimiento del PIB en América Latina durante el periodo estudiado ha variado notablemente, es así que en el año 2000 la tasa de crecimiento del PIB creció en 11\% debido a una mejora en el mercado laboral de la región, para el periodo entre (2001-2002) presento una caída con una variación promedio del -6,99\% debido a la crisis económica de Argentina, por otra parte, se puede observar que en el periodo comprendido por el año 2003 al año 2008 existió un crecimiento económico significativo con una variación promedio del15,5\%.

A finales del año 2008 se produjo la crisis económica mundial, teniendo origen en Estados Unidos a causa del colapso de la burbuja inmobiliaria en el 2006, lo cual afectó directamente a la economía de los países que conforman América Latina, por tal motivo en el año 2009 se ve un decrecimiento económico del 6,17\% con 
relación al año anterior, mientras que para el 2010 los países latinoamericanos al incentivar el consumo y la inversión, es decir dinamizando la demanda interna tuvieron un incremento en el PIB en $25 \%$, esto a su vez ayudó a la recuperación de las economías lo cual se ve reflejado hasta el año 2014.

Por otra parte, se evidencia un decrecimiento en la economía de América Latina para el año 2015 en 14,02\% y para el año 2016 con una caída del 1,70\% motivo de la disminución del consumo interno y la baja inversión, acompañado de las bajas exportaciones netas y en consecuencia a los problemas presentados por decisiones económicas y políticas. Con respecto al año 2017 existió un crecimiento económico del 10,82\%, favorecido por el notable crecimiento económico mundial principalmente de las economías desarrolladas y en desarrollo, a su vez el aumento en el comercio mundial y la recuperación de los precios de los productos básicos han sido factores importantes para la recuperación del crecimiento económico en Latinoamérica.

\subsection{Inflación}

La tasa de inflación en América Latina ha presentado fluctuaciones considerables durante el periodo de estudio, este comportamiento ha sido perjudicial para la economía de cada país.

\section{Gráfico 3}

Tasa de Inflación en los países de Latinoamérica

Período 2000 - 2017, (valores promedios y porcentajes)

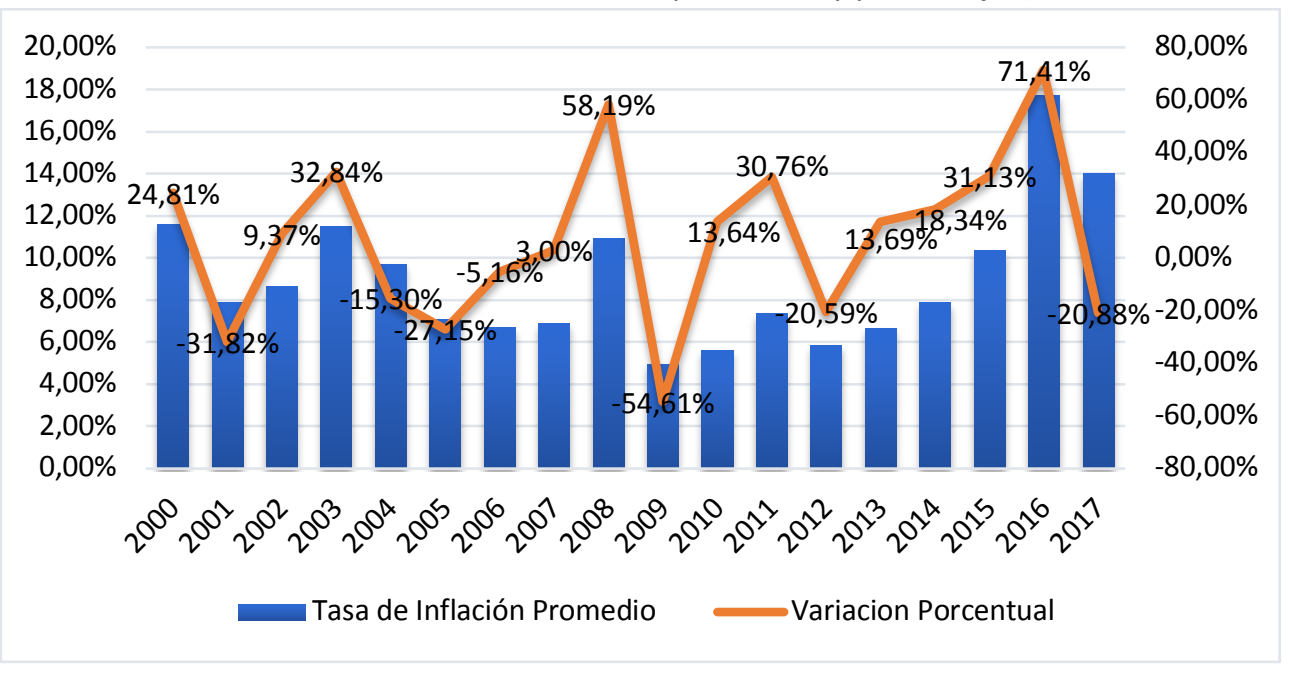

Fuente: Elaboración propia con base en datos del Banco Mundial (2019)

En el Gráfico 3 se puede observar que la tasa de inflación ha fluctuado constantemente durante todo el periodo estudiado, teniendo tendencias de crecimiento y decrecimiento. En el año 2000 se puede evidenciar una tasa de inflación alta en la región, siendo Ecuador el país con mayor nivel de inflación con un 96\%, a causa de la dolarización y el feriado bancario en el año anterior, para el año 2001 estos niveles se redujeron a un 7,90\% en comparación al año 2000, sin embargo se ve una tendencia creciente desde el año 2001 al año 2003 llegando a un máximo de 11,47\% en la tasa de inflación de la región.

Por otra parte, para el año 2004 hasta el año 2006 se evidencia una reducción en la inflación regional del 9,72\% y 6,71\% en relación a los años anteriores, en donde Chile, Perú y Panamá son los países con menor inflación, mientras que Republica Dominicana, Haití y Venezuela son los países con mayor inflación de la región. Para el año 2008 se incrementa notablemente la inflación a un 8,2\% en América Latina, siendo Venezuela el país con la mayor tasa de inflación con el 30,40\% y México con la menor tasa del 5,12\%. 
Para los años 2010 y 2011 los valores de la inflación aumentaron en 5,64\% y 7,38\% correspondientemente, este incremento se dio en la mayoría de países de la región en excepción de Ecuador ya que presenta disminución en la tasa de inflación a comparación de los años anteriores, mientras que Venezuela presenta una tendencia creciente. En el año 2012 al año 2016 los niveles de inflación se incrementaron notablemente del 5,86\% al $17,72 \%$, esto en consecuencia del alza en los precios alimenticios y bebidas en la región, para el 2017 se observa una reducción de la inflación al 14,02\% en la región, teniendo a Venezuela como el país con mayor tasa de inflación desde el año 2008 al año 2017 a causa del desabastecimiento de productos de primera necesidad y el exceso de circulación monetaria en el país.

\subsection{Tasa de Desempleo}

Con base en la información que se ha recabado, se puede observar el comportamiento de la tasa de desempleo en Latinoamérica, como muestra el gráfico 4, la tasa de desempleo maneja una tendencia al decrecimiento en el periodo comprendido entre (2000-2012) a excepción de los países de Haití, Honduras y México en donde se registra un comportamiento contrario sin embargo es preciso mencionar que a partir del año 2013 hasta el 2017 se registra cierta tendencia mínima al incremento.

Gráfico 4

Tasa de Desempleo en los países de Latinoamérica

Período 2000 - 2017, (en promedio porcentual)

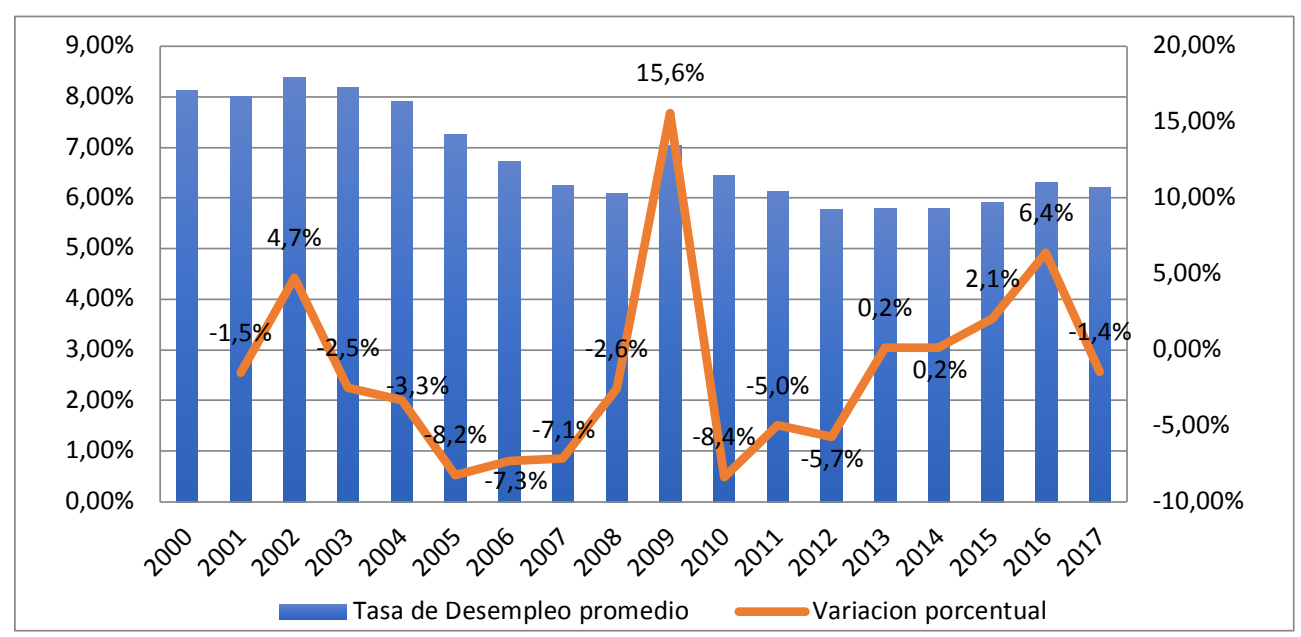

Fuente: Elaboración propia con base en datos del Banco Mundial (2019)

Sin embargo, es preciso mencionar el año 2002 puesto que muestra los mayores porcentajes en la tasa de desempleo para los primeros años de estudio al igual que el año 2003 aunque tuvo un leve decrecimiento al año anterior. Mientras que para el periodo entre (2004 - 2008) existió un decrecimiento con una variación porcentual del $-5,7 \%$, esto se debió a la dinamización económica que se presentó en el período. Por otra parte, en el año 2009 la tasa de desempleo creció notablemente en 15,6\% en relación al año anterior ya que esto se dio por la crisis económica que afecto a todo el sector económico y social en la región.

Para el periodo entre (2010-2017) se evidencio un decrecimiento en la tasa de desempleo en comparación al 2009, ya que se vio un notable crecimiento económico en la región, lo cual motiva a la dinamización en los diferentes sectores económicos y a su vez una inflación decreciente en los países de América del sur motivo a al consumo y por ende a la producción, creando así fuentes de empleo (CEPAL, 2018). 
Además, se considera necesario el analizar de manera profunda el caso de Haití y Colombia que son las naciones que manejan las más altas tasas de desempleo a lo largo del periodo en estudio, y el caso de Bolivia y Panamá que generan tasas de desempleo más bajas.

Para el caso especial de Haití se registra una tasa de desempleo en el año 2000 del $8.34 \%$, sin embargo para el año 2009 muestra su más alto porcentaje a lo largo del periodo de estudio puesto que registra el 16.24\% y finaliza con cierto decrecimiento para el año 2017 con el 13.40\%; por lo tanto si bien la tendencia disminuye su comportamiento general es siempre al incremento, por tal razón este país maneja las más altas de desempleo de Latinoamérica, en el caso de Colombia esta registra una conducta similar puesto que su mayor valor porcentual para el año 2000 con el 20.54\%, y si bien muestra una tendencia a decrecer para el año 2017 genera el $8.84 \%$, provocando que este país sea la segunda nación con altos porcentajes en el manejo de la tasa de desempleo.

No obstante, para el país boliviano la tasa de desempleo ha sido manejada de manera estable y equilibrada puesto que oscila entre el rango del $3.50 \%$ al $2.01 \%$, teniendo variaciones mínimas entre el periodo de estudio, es así que para el año 2000 muestra un valor de 3.37\% y finaliza el año 2017 con el 3.23\%. En el caso de Panamá la situación es muy parecida a su similar de la nación boliviana ya que maneja un rango estable del $4.55 \%$ al $2.31 \%$, este hecho se consolida al revisar el valor porcentual del año 2000 donde registra el $4.21 \%$ y en el año 2017 donde marca un $3.90 \%$ en la tasa de desempleo.

\subsection{Ingreso Nacional Bruto per cápita}

El Ingreso Nacional Bruto per cápita ha presentado un acrecentamiento, con tendencia al aumento lo cual favorece al crecimiento económico puesto que incentiva ciertas variables como el empleo que actúa de manera positiva hacia la prosperidad económica.

Gráfico 5

Ingreso Nacional Bruto per cápita en los países de Latinoamérica Período 2000 - 2017, (en miles de dólares y porcentajes)

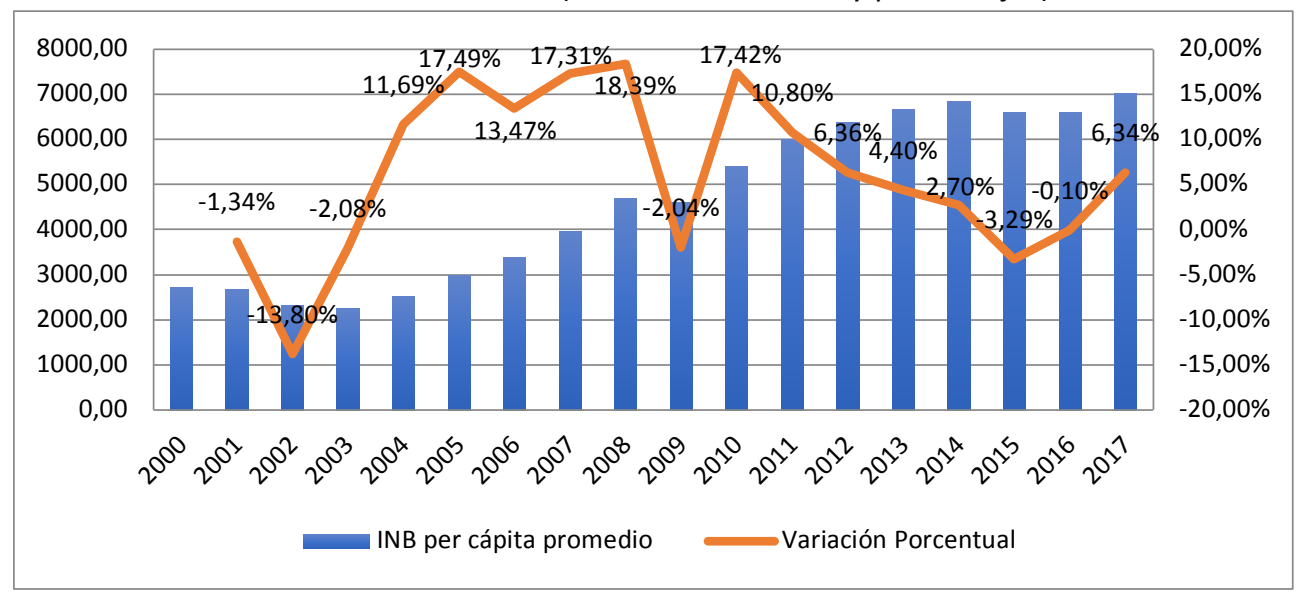

Fuente: Elaboración propia con base en datos del Banco Mundial (2019)

Con base en la información obtenida, se puede observar en el Gráfico 5 el aumento del ingreso nacional bruto per cápita en todos los países de Latinoamérica; puesto que desprende su análisis para el año 2001 con una variación porcentual de-1,34\% con relación al año anterior y finaliza en el año 2017 con una variación porcentual de 6,34\%. Con base al gráfico presentado podemos evidenciar que existe un escenario de crecimiento, por tanto, los países de Uruguay y Chile son los que mayor participación positiva evidencian; sin embargo, caso contrario se denota un decrecimiento notable para Haití y Bolivia. 
En el caso de Uruguay se refleja una tendencia marcada al aumento, pero vale notar que su cúspide fue en el año 2013 puesto que muestra un valor de 14.646 .02 miles de dólares, sin embargo, el año menos favorable a esta nación fue el año 2003 ya que marca apenas 3.147.11 miles de dólares. Un caso parecido muestra la nación chilena donde su año más destacado fue el 2013 con 12.054.20 miles de dólares no obstante el año 2002 es el que registra menos intención con un valor de 3.586.20 miles de dólares (Ver anexo 5).

Con lo que respecta al país haitiano y boliviano estos manejan valores de 538.68 y 1513.58 miles de dólares respectivamente en promedio; Haití, si bien refleja un mínimo crecimiento en el periodo analizado, está considerado como el país que más bajo ingreso nacional bruto per cápita maneja y es así que para el año 2017 genera apenas 718.26 miles de dólares; finalmente en el caso de Bolivia este país maneja una tendencia al aumento que varía de manera constante, esto es evidente en el análisis del periodo determinado, ya que para el año 2000 maneja un valor de 868.12 miles de dólares, en el año 2008 muestra 1368.67 miles de dólares y culmina la etapa de estudio en el año 2017 con 2.664 .17 miles de dólares.

\subsection{Estimación del Modelo Econométrico}

Se procede a estimar el modelo econométrico para medir el impacto de las variables macroeconómicas en la inversión extranjera directa, aplicando el método de mínimos cuadrados generalizados mediante datos de panel, ya que se cuenta con una dimensión transversal de 19 países de Latinoamérica para un período de 17 años, es decir se posee 323 observaciones.

Tabla 2

Regresión mediante Mínimos Cuadrados Generalizados por datos de panel

\begin{tabular}{lcc}
\hline Variable Dependiente & \multicolumn{2}{c}{ LOGIED } \\
\hline Variable Independiente & Coeficiente & Prob. \\
\hline LOGIED(-1) & 0.808311 & 0.0000 \\
LOGTCPIB & 0.114272 & 0.0092 \\
LOGTINFLACION & -0.034327 & 0.4997 \\
LOGTDESEMPEO & 0.089476 & 0.3231 \\
LOGINBPC & 0.259836 & 0.0011 \\
C & 1.715 .423 & 0.0026 \\
\hline R-cuadrado & 0.847638 \\
\hline F-estadístico & 2.648140 \\
\hline Criterio de información de Akaike & 2.021236 \\
\hline Durbin-Watson & \multicolumn{2}{c}{2.494157} \\
\hline Prob (F-estadístico) & \multicolumn{2}{c}{0.000000} \\
\hline
\end{tabular}

Fuente: Elaboración propia con base en EVIEWS 10

Se observa que las variables independientes tienen relación directa e inversa respecto a la variable dependiente, siendo directas la LogIED-1, LogTCPIB y LogINBpc con un coeficiente positivo cumpliendo con la teoría económica y a su vez son significativas con el modelo, mientras que LogTInflación es inversa con la IED ya que presenta un coeficiente negativo pero no es significativa con el modelo. Por otro lado, se puede observar que LogTDesempleo presenta un coeficiente positivo lo cual no cumple con la teoría y no es significativo con el modelo.Se procede a aplicar el Test de Hausman para establecer si existen diferencias significativas entre las medias de las variables de los modelos estáticos aplicados, es decir si se utilizara el modelo de efectos fijos o el modelo de efectos variables: 


$$
\begin{gathered}
\boldsymbol{H}_{0}>0,05=\text { Se asume Efectos Aleatorios } \\
\boldsymbol{H}_{\mathbf{1}}<0,05=\text { Se asume Efectos Fijos }
\end{gathered}
$$

Tabla 3

Test de Hausman

\begin{tabular}{lllll}
\hline \multicolumn{2}{l}{ Efectos aleatorios correlacionados - Hausman Test } \\
Resumen de Prueba & & Chi-Sq. Estadístico & Chi-Sq. d.f. & Prob. \\
\hline Sección Transversal aleatoria & & 150.886455 & 5 & 0.0000 \\
\hline Variable & Fijo & Aleatorio & Var (Dif.) & Prob. \\
\hline LOGIED(-1) & 0.166187 & 0.808311 & 0.002778 & 0.0000 \\
LOGTCPIB & 0.078398 & 0.114272 & 0.000221 & 0.0159 \\
LOGINBPC & 1.159 .490 & 0.259836 & 0.015084 & 0.0000 \\
LOGTINFLACION & 0.048263 & -0.034327 & 0.001367 & 0.0255 \\
LOGTDESEMPEO & 0.206384 & 0.089476 & 0.037290 & 0.5449 \\
\hline
\end{tabular}

Fuente: Elaboración propia con base en EVIEWS 10

En la tabla 2 se puede observar que al aplicar el Test de Hausman arrojó resultados que representa evidencia en contra de la hipótesis nula, por lo tanto se debe estimar un modelo de efectos fijos, Los resultados de la estimación del modelo de efectos fijos se presenta en la tabla 4.

Tabla 4

Modelo de Efectos Fijos

\begin{tabular}{lll}
\hline Variable Dependiente: LOGIED & & \\
\hline Variable Independiente & Coeficiente & Prob. \\
\hline LOGIED(-1) & 0.169297 & 0.0046 \\
LOGTCPIB & 0.080902 & 0.0237 \\
LOGINBPC & 1.083962 & 0.0000 \\
C & 8.641621 & 0.0000 \\
\hline R-cuadrado & 0.913625 & \\
\hline F-estadístico & 1.133290 & \\
\hline Criterio de Información de Akaike & 1.590078 & \\
\hline Durbin-Watson & 2.013385 & \\
\hline Prob(F-estadístico) & 0.000000 & \\
\hline
\end{tabular}

Fuente: Elaboración propia con base en EVIEWS 10

En donde la ecuación sería:

$\operatorname{LogIED}_{i t}=8,641+0,1692 \operatorname{LogIED}_{i t-1}+0,0809 \operatorname{LogTcPIB}_{1 i t}+1,0839_{4} \operatorname{LogINBp}_{4 i t}+\mu_{i t}$

Estableciendo que:

La relación existente entre la IED rezagada en un período con la IED actual es de 0,1692. Es decir que si se incrementa $1 \%$ en la IED rezagada, la inversión extranjera directa actual incrementa en $0,16 \%$. Por otra parte, si la tasa de crecimiento del PIB aumenta en 1\% la IED aumentará en un 0,08\%. Así mismo si el ingreso nacional bruto per cápita aumenta en $1 \%$ la inversión extranjera directa aumentará en 1,08\%.

El Coeficiente de determinación R-cuadrado $\left(\mathrm{R}^{2}\right)$ establece la proporción en la que las variables independientes contribuyen a explicar la variable dependiente, obteniendo en el modelo un coeficiente de 0.91 , lo que permite determinar que las variables propuestas se ajustan en un $91 \%$ a la variable dependiente IED. 


\section{Conclusiones}

La Inversión Extranjera Directa de los países Latinoamericanos presenta notables variaciones durante el periodo 2000-2017 con una variación porcentual promedio del 7,34\%, teniendo mayor colocación de capitales en el sector de recursos naturales, por lo cual los países que reciben mayor inversión son Brasil, México, Chile y Colombia representando el $80,51 \%$ del total promedio de los flujos de IED en la región, mientras que los países con menor flujo de IED son Bolivia, Ecuador, El Salvador, Guatemala, Haití, Nicaragua y Paraguay representando apenas el 2,44\% del total promedio de IED en la región.

Mediante la aplicación del modelo econométrico se pudo comprobar que las variables consideradas como determinantes de la IED fueron significativas tanto como la IED rezagada en un periodo, la tasa de crecimiento y el INB per cápita, mientras que las variables Tasa de Inflación y la Tasa de Desempleo presentaron un nivel de significancia mayor a 0,05 por lo cual no son significativa para el modelo. Es así que se determinó que la IED se ve motivada principalmente por el INB per cápita y por el crecimiento económico ya que estos ayudan a la atracción de capitales lo cual es beneficioso para el país receptor.

\section{Referencias bibliográficas}

Banco Mundial. (2009). El Organismo Multilateral de Garantías para Inversiones. Obtenido de Banco Mundial: http://www3.icex.es/icex/cma/contentTypes/common/records/mostrarDocumento/?doc=4620228

Cabrera, M. J., \& Naranjo , S. (2018). Inversión Extranjera Directa como Motor de Desarrollo Económico. Obtenido de Desde el Centro: Conectando ideas para la Productividad: http://www.desdelcentro.org/inversion-extranjera-directa-motor-desarrollo-economico/

CEPAL. (2004). La Inversión Extranjera Directa en América Latina y el Caribe, 2003. Obtenido de Comisión Económica para América Latina y el Caribe: https://www.cepal.org/es/publicaciones/1126-la-inversionextranjera-america-latina-caribe-2003

CEPAL. (2009). La Inversión Extranjera Directa en América Latina y el Caribe, 2008. Obtenido de Comisión Económica para América Latina y el Caribe: https://www.cepal.org/es/publicaciones/1138-la-inversionextranjera-directa-america-latina-caribe-2008

CEPAL. (2010). La Inversión Extranjera Directa en América Latina y el Caribe, 2009. Obtenido de Comisión Económica para América Latina y el Caribe: https://www.cepal.org/es/publicaciones/1140-la-inversionextranjera-directa-america-latina-caribe-2009

CEPAL. (2012). La Inversión Extranjera Directa en America Latina y el Caribe 2011. Obtenido de Comisión Económica para América Latina y el Caribe: https://repositorio.cepal.org/bitstream/handle/11362/1146/S1200384_es.pdf?sequence=1\&isAllowed=y

CEPAL. (2016). La Inversión Extranjera Directa en América Latina y el Caribe, 2016. Obtenido de Comisión Económica pa América Latina y el Caribe: https://www.cepal.org/es/publicaciones/40213-la-inversionextranjera-directa-america-latina-caribe-2016

CEPAL. (2017). La Inversión Extranjera Directa de América Latina y el Caribe, 2017. Obtenido de Comisión Económica de América Latina y el Caribe: https://www.cepal.org/es/publicaciones/42023-la-inversionextranjera-directa-america-latina-caribe-2017

CEPAL. (2018). Estudio Económico de América Latina y el Caribe. Evolución de la inversión en América Latina y el Caribe: hechos estilizados, determinantes y desafíos de política. Obtenido de Comisión Económica para 
América Latina y el Caribe: cepal.org/es/publicaciones/43964-estudio-economico-america-latina-caribe2018-evolucion-la-inversion-america

CEPAL. (2018). La Inversión Extranjera Directa en América Latina y el Caribe, 2018. Obtenido de Comisión Económica para América Latina y el Caribe: https://www.cepal.org/es/publicaciones/43689-la-inversionextranjera-directa-america-latina-caribe-2018

Chávez, D., \& Félix, J. (2003). México, opción para el inversionista extranjero. Recuperado el 19 de Junio de 2019, de Bibliotecas Universidad de las Américas Puebla: http://catarina.udlap.mx/u_dl_a/tales/documentos/lcp/chavez_I_dd/

Díaz, R. (2003). Las teorías de la localización de la inversión extranjera directa: una aproximación. Revista Galega de Economía, pp. 8.

Dunning, J. H. (1977). "Trade, Location of Economic Activity and the Multinational Enterprise: A Search for an Eclectic Approach". London: Macmillan.

Dunning, J. H. (1988). The eclectic paradigm of international production: a restatement and some posible extensions., 19(1). Journal on International Businees Studies.

Espín, J. A., Córdova, A. C., \& López, G. E. (2016). Inversión extranjera directa: su incidencia en la tasa de empleo del Ecuador. Retos, 217.

Flora, P., \& Agrawal, G. (2014). Determinants of Direct Foreign Investment as a Means of International Market Entry: A Review. Indian Journal of Economics and Development.

Gil , E. A., López, S. F., \& Espinosa, D. A. (2013). Factores determinantes de la inversión extranjera directa en América del Sur. Universidad de Antioquia.

Gobierno de México. (2013). Durante 2012, con cifras preliminares, México registró 12,659.4 millones de dólares de Inversión Extranjera Directa. Obtenido de Secretaría de Economía:

https://www.gob.mx/se/prensa/durante-2012-con-cifras-preliminares-mexico-registro-12-659-4-millonesde-dolares-de-inversion-extranjera-directa

Goto , B., Lagos , M., \& Spencer, E. (2016). Estudio de factores que influyen en los flujos de inversión extranjera directa hacia América Latina. Obtenido de Repositorio de la Universidad de Chile:

repositorio.uchile.cl/handle/2250/139725

Jiménez, D. E., \& Rendón, H. (2012). Determinantes y efectos de la Inversión Extranjera Directa: revisión de literatura. Departamento de Economia de la Facultad de Ciencias Humanas y Económicas. Medellín: Universidad Nacional de Colombia. Obtenido de https://dialnet.unirioja.es/servlet/articulo?codigo=6266761

Mogrovejo, J. (2005). Factores determinantes de la Inversión Extranjera Directa en algunos países de Latinoamérica. Estudios Económicos de Desarrollo Internacional, 5, 2. Santiago de Compostela, La Coruña, España. Obtenido de Universidade de Santiago de Compostela: http://www.usc.es/economet/journals/eedi/eedi524.pdf

Observatorio de Multinacionales en América Latina. (2012). Inversión Extranjera Directa. Obtenido de Investigación, documentación y denuncia de los impactos de las multinacionales españolas en América Latina: http://omal.info/spip.php?article4822 
Ormeño, C. L., Zambrano, M., \& Solorzano, G. Análisis de la Inversión Extranjera Directa de los Países de América Latina ¿Cuáles son sus determinantes? Un estudio en Datos de Panel (1999 - 2010). Escuela Superior Politécnica del Litoral, Ecuador.

Ozawa, T. (1992). "Foreign Direct Investment and Economic Development" (Vol. I). Transnational Corporations.

Penfold, M., \& Curbelo, J. L. (2013). Hacia una nueva agenda en inversión extranjera directa. Tendencias y realidades en América Latina. Cyngular. Obtenido de publicaciones.caf.com/media/33311/inversionextranjeradirecta.pdf

Rodríguez, G., \& Forero, D. Factores determinantes de la Inversión Extranjera Directa. Universidad Tecnológica de Bolivar, Colombia.

Saravia, A. (2009). "Determinantes de la Inversión Extranjera Directa en Bolivia 1996 - 2008" - El impacto en los $R R N N$. Cochabamba: Universidad Mayor de San Simón.

Vernon, R. (1966). International Investment and International Trade in the Product Life Cycle. Revista: Quarterly Journal of Economics.

\footnotetext{
Esta obra está bajo una Licencia Creative Commons Attribución-NoCommercial 4.0 International

\section{(cc) BY-NC}

